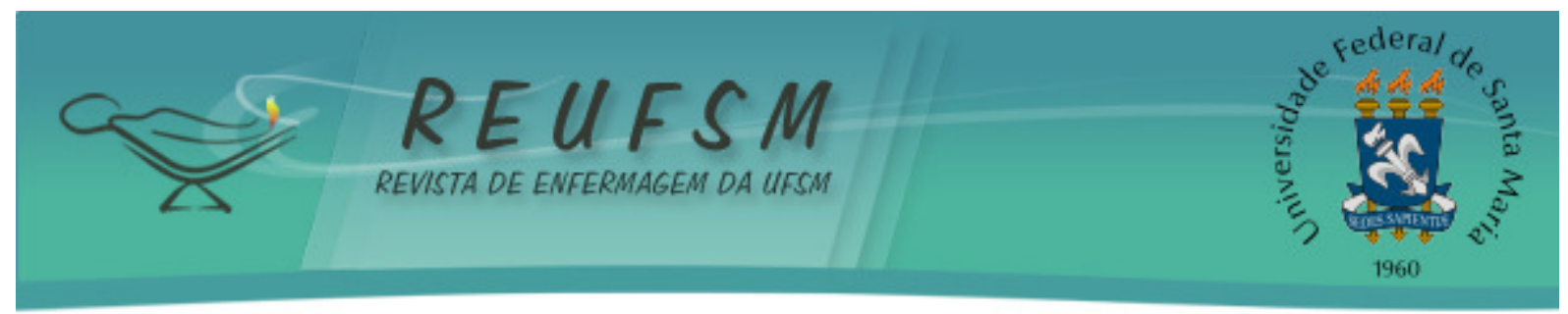

ARTIGO ORIGINAL

\title{
DIFERENÇAS NA AMPLITUDE LATERAL DO MOVIMENTO DA COLUNA CERVICAL ENTRE JOVENS E IDOSOS
}

\section{DIFFERENCES IN THE LATERAL RANGE OF MOTION OF THE CERVICAL SPINE BETWEEN YOUNG AND ELDERLY ADULTS}

\section{DIFERENCIAS EN LA AMPLITUD LATERAL DEL MOVIMIENTO DE LA COLUMNA CERVICAL ENTRE JÓVENES Y ANCIANOS}

\author{
Anna Cláudia Freire de Araújo Patrício ${ }^{1}$ \\ Karoline de Lima Alves ${ }^{2}$ \\ Jiovana de Souza Santos ${ }^{3}$ \\ Daniele Ferreira Rodrigues ${ }^{4}$ \\ Teresa Cristina Rosa Romero Navarine ${ }^{5}$ \\ Michelle Salles Barros de Aguiar ${ }^{6}$
}

Doi: 10.5902/2179769211310

RESUMO: Objetivo: analisar as diferenças na amplitude lateral do movimento da coluna cervical, entre jovens e idosos. Método: estudo quantitativo e comparativo, em João Pessoa/Paraíba, com 55 idosos e 55 jovens. Realizou-se teste de flexibilidade da coluna cervical com aparelho goniômetro Sanny ${ }^{\circledR}$. Considerou-se $p<0,05$. Resultados: idosos tinham $68 \pm 6$ anos e jovens $23 \pm 3$ anos, apresentaram $20,4 \pm 6,4$ graus de amplitude lateral direita e $31 \pm 7$ graus, respectivamente. No lado esquerdo, os idosos atingiram $29 \pm 7$ graus e os jovens $32 \pm 6$ graus. Ambos os lados com $p<0,05$. Conclusões: observou-se que os sujeitos dos grupos apresentaram amplitude inferior ao estabelecido na literatura. É necessário implantar métodos que avaliem indivíduos a fim de detectar precocemente a amplitude da coluna cervical diminuída.

Descritores: Amplitude de movimento articular; Atlas cervical; Idoso; Adulto jovem; Enfermagem.

ABSTRACT: Aims: to analyze the differences in the lateral range of motion of the cervical spine, among young and elderly people. Method: a quantitative and comparative study carried out in João Pessoa/Paraiba, with 55 elderly people and 55 young people. A flexibility test of the cervical spine was held, and the goniometer Sanny $\otimes$ was employed. It was considered $p<0.05$. Results: the elderly were $68 \pm 6$ years old and the young $23 \pm 3$ years old, and they showed $20.4 \pm 6.4$ degrees and $31 \pm 7$ degrees of right range of motion, respectively. Regarding the left side, the elderly reached $29 \pm 7$ degrees and the young $32 \pm 6$ degrees; both sides with $p<0.05$. Conclusions: it was observed that the

\footnotetext{
${ }^{1}$ Enfermeira. Mestranda em Enfermagem. Programa de Pós Graduação em Enfermagem da Universidade Federal da Paraíba(UFPB). Integrante do Grupo de Estudos e Pesquisa em Envelhecimento e Representações Sociais/UFPB.João Pessoa/PB,Brasil. E-mail:claudia.freirearaujo@gmail.com

${ }^{2}$ Enfermeira. Mestranda em Enfermagem. Programa de Pós Graduação em Enfermagem da Universidade Federal da Paraíba(UFPB). Integrante do Grupo de Estudos e Pesquisa em Envelhecimento e Representações Sociais/UFPB.João Pessoa/PB,Brasil. E-mail:krol_lima_17@hotmail.com

${ }^{3}$ Graduanda de Enfermagem. Centro Universitário de João Pessoa/PB. Integrante do Grupo de Estudos e Pesquisa em Envelhecimento e Representações Sociais/UFPB. João Pessoa/PB,Brasil. Email:jiovana_santos@hotmail.com

${ }^{4}$ Fisioterapeuta. Mestre em Saúde Coletiva.Universidade Federal de Pernambuco(UFPE).Recife/PE,Brasil. Email:daniele-fisio@hotmail.com

${ }^{5}$ Enfermeira. Mestranda em Enfermagem.Programa de Pós Graduação em Enfermagem da Universidade Federal da Paraíba(UFPB).João Pessoa/PB,Brasil. E-mail:teresanavarine@hotmail.com

${ }^{6}$ Química Industrial. Mestre em Engenharia de Produção. Universidade Federal da Paraíba. João Pessoa/PB,Brasil. E-mail: michelleestatistica@gmail.com
} 


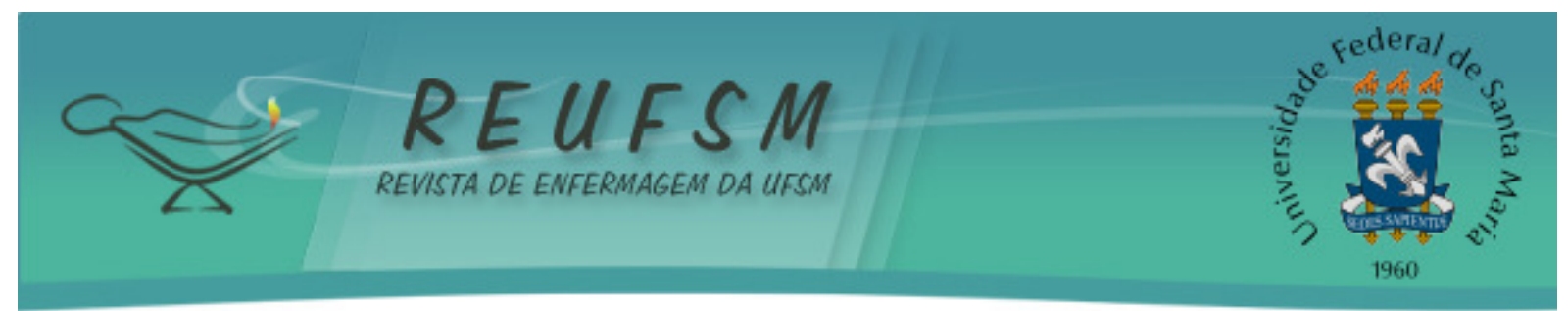

participants showed lower range of motion, compared to the range established in the literature. It is necessary to develop methods that evaluate individuals in order to detect limited range of motion of the cervical spine early on.

Descriptors: Range of motion, articular; Cervical atlas; Aged; Young adult; Nursing.

RESUMEN: Objetivo: analizar las diferencias en la amplitud lateral del movimiento de la columna cervical, entre jóvenes y ancianos. Método: estudio cuantitativo y comparativo, hecho en João Pessoa/Paraíba, con 55 ancianos y 55 jóvenes. Se realizó prueba de flexibilidad de la espina dorsal cervical con aparato goniómetro Sanny®. Se consideró $p<$ 0 ,05. Resultados: los ancianos tenían $68 \pm 6$ años y los jóvenes $23 \pm 3$ años, presentaron $20,4 \pm 6,4$ grados de amplitud lateral derecha y $31 \pm 7$ grados, respectivamente. En el lado izquierdo, los ancianos alcanzaron $29 \pm 7$ grados y los jóvenes $32 \pm 6$ grados. Ambos lados con $p<0.05$. Conclusiones: se observó que los sujetos de los grupos mostraron menor amplitud que la establecida en la literatura. Es necesario implementar métodos que evalúen individuos con el fin de la detección precoz de la amplitud de la espina dorsal cervical disminuida.

Descriptores: Rango del movimiento articular;Atlas cervical; Anciano; Adulto joven; Enfermería.

\section{INTRODUÇÃO}

A coluna vertebral é constituída por um tecido de alta especialização e fragilidade por onde passam todas as ordens sensitivas e motoras que vão da periferia para o cérebro e vice-versa. Tal estrutura, se afetada, implicará danos sérios a saúde do ser humano podendo adquirir doenças irreversíveis que irão influenciar na sua vida, impedindo-o de exercer suas atividades normalmente. ${ }^{1}$ No idoso, a prevalência de mortes associada a doenças do sistema osteomuscular apresenta-se em índices elevados conforme pesquisa realizada no Paraná em 2010. ${ }^{2}$

A literatura aponta que a coluna cervical possui maior mobilidade quando comparada com outras áreas da coluna vertebral devido às diferenças anatômicas. A região cervical possui graus de liberdade, realizando movimentos de rotação com cerca de $90^{\circ}$ e de lateralização $45^{\circ}$, para cada lado. ${ }^{3}$

A disfunção somática vertebral caracteriza-se pela hipomobilidade vertebral, ou seja, uma vértebra não está livre para fazer seu movimento em relação à outra vértebra e ao disco intervertebral. Esta alteração ocorre por espasmos dos músculos intertransversários, multífidos e rotadores que mantêm firme a articulação interfacetária ${ }^{4}$, podendo repercutir através de tensões miofasciais nas articulações temporomandibulares (ATM) e ossos do crânio. ${ }^{5}$

A hipomobilidade pode ser mensurada pela Avaliação da Amplitude de Movimento (ADM) e consiste em um importante instrumento para diagnosticar disfunções musculoesqueléticas, analisar a progressão das doenças, avaliar o efeito de diferentes tratamentos e acompanhar a evolução do paciente durante a reabilitação. ${ }^{5}$

A mensuração da ADM é de fácil aplicabilidade e possibilita achados clinicamente precisos, contudo é necessária a avaliação por métodos e ferramentas confiáveis, não invasivos e que possam ser utilizadas por diferentes avaliadores. ${ }^{6}$

Há uma grande variedade de métodos não invasivos disponíveis para verificar a ADM, como: a estimativa visual; o goniômetro; o inclinômetro; o potenciômetro; tecnologias de bússolas; de vídeo e eletromagnéticas. ${ }^{7}$ Neste estudo optou-se pelo goniômetro por ser um instrumento de medição ou de verificação de medidas angulares. 


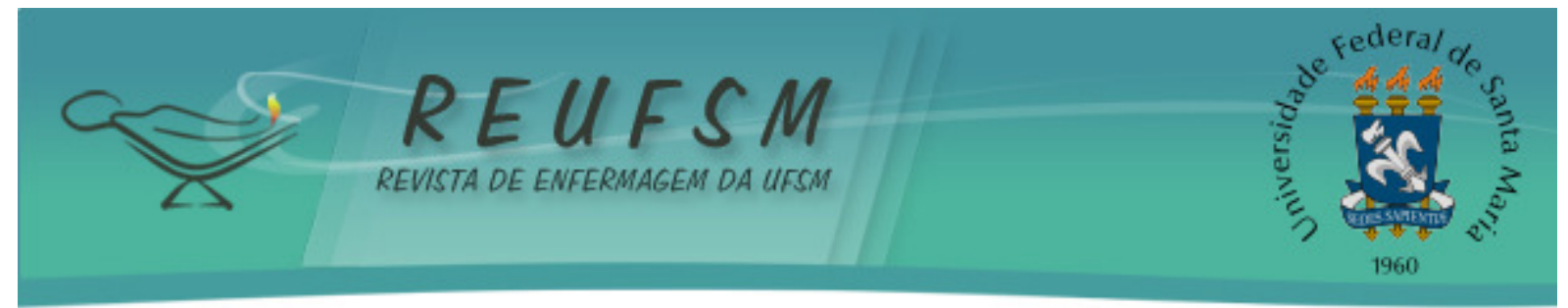

A coluna cervical responsabiliza-se por diversas funções no corpo humano, como o comando de movimentos e sensações que podem ser prejudicadas por lesões ou pelo desgaste devido ao sofrimento mecânico diário à qual é submetida.

Os distúrbios musculoesqueléticos e o próprio envelhecimento se relacionam a diminuição da amplitude de movimento da coluna cervical, tornando relevante a realização de estudos comparativos entre jovens e idosos, ainda escassos na literatura. Estudo $^{8}$ realizado em Londrina no ano de 2011 revelou que a diminuição da ADM em idosos é significativa e pode estar correlacionada a cervicalgia e zumbido.

Sendo assim, o conhecimento prévio das mudanças provenientes do processo de envelhecimento, suas implicações clínicas e funcionais darão suporte aos profissionais de saúde para avaliar, tratar e, principalmente, promover programas preventivos a fim de minimizar os efeitos das disfunções cinésio-funcionais em idosos, resgatando sua autonomia funcional e melhorando sua qualidade de vida. ${ }^{9}$

Nesse contexto, surgiu o questionamento: Quais as diferenças de amplitude lateral do movimento da coluna cervical entre jovens e idosos? Tal conhecimento pode ser útil para a equipe de saúde multiprofissional, incluindo os enfermeiros, no que concerne ao embasamento científico, auxiliando no diagnóstico precoce e tomadas de decisões mais eficazes, promovendo a reabilitação de pacientes com comprometimento da ADM. Diante disso, esse estudo teve como objetivo analisar as diferenças na amplitude lateral do movimento da coluna cervical entre jovens e idosos.

\section{MÉTODO}

Trata-se de um estudo comparativo, de natureza descritiva e abordagem quantitativa, realizado no município de João Pessoa, Paraíba (PB), Brasil, região do Nordeste brasileiro, oriundo de um recorte do projeto intitulado: Espaço Morto em Prancha Longa de Imobilização Cervical: um achado relevante para adequação tecnológica, possuindo como objetivo específico analisar as diferenças na amplitude lateral do movimento cervical entre jovens e idosos.

Participaram do estudo dois grupos, sendo um de jovens (GJ) e outro de idosos (GI). A população do grupo jovem foi composta por 200 sujeitos universitários matriculados no curso de enfermagem de uma instituição de ensino superior na cidade de João Pessoa/PB. A população idosa, constituída por 180 voluntários, participantes das atividades de um Grupo de Idosos do Clube da Pessoa Idosa, da Prefeitura de João Pessoa/PB.

Para definir o quantitativo de sujeitos para compreender a amostra, realizou-se cálculo amostral com auxílio do Programa Statdisk versão 11.1.0 USA. Para os grupos jovem e idoso utilizou-se nível de confiança de $95 \%$ e margem de erro de $11 \%$, obtendo uma amostra de 55 indivíduos para cada grupo.Totalizando 110 participantes do estudo.

0 critério de inclusão adotado para o GJ foi possuir idade entre 20 e 30 anos. No GI a idade dos sujeitos deveria ser igual ou superior a 60 anos e possuir capacidade cognitiva preservada.

O envelhecimento populacional na cidade de João Pessoa, considerada pelo Instituto Brasileiro de Geografia e Estatística (IBGE) no ano $2010^{10}$ como a terceira com maior número de idosos no país e a primeira na região Nordeste, tornando-se essencial conhecer a realidade desta população nos mais diversos setores da saúde, neste estudo, na amplitude do movimento.

Os dados foram coletados durante os meses de maio e junho do ano 2011 e se deu por meio do teste de flexibilidade da coluna cervical, mensurado através do aparelho goniômetro da marca Sanny ${ }^{\circledR}$ que tem por finalidade verificar medidas angulares. 


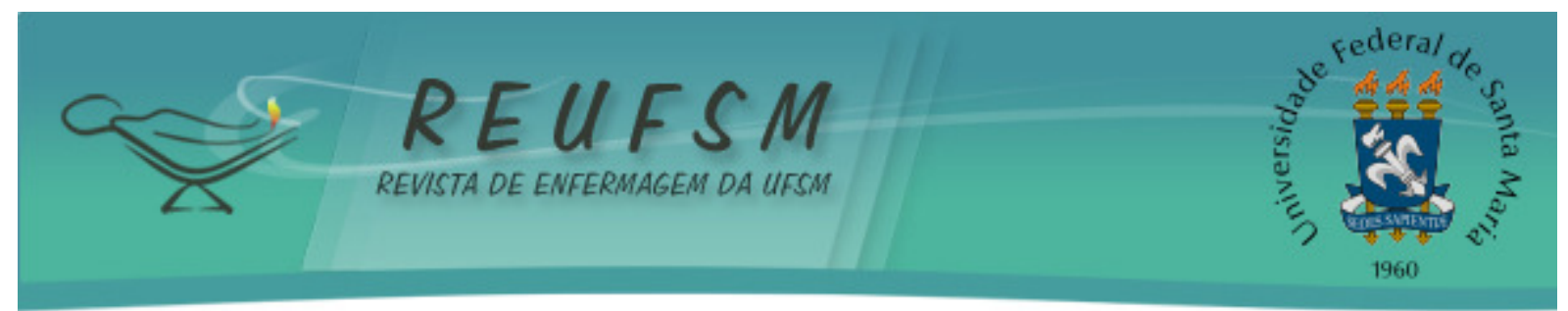

Para avaliação da ADM da coluna cervical solicitou-se que o sujeito lateralizasse a cabeça em direção ao acrômio da escápula, ocasião em que o pesquisador registrou o grau de flexibilidade com o auxílio do aparelho de acordo com o método proposto por Peeler e Anderson $^{11}$. O equipamento goniômetro foi colocado na porção ântero-posterior do pescoço. O sujeito em posição sentado, mãos sobre as coxas, joelho e tornozelo a $90^{\circ}$ graus de flexão com os pés apoiados no chão. Posteriormente, apresentou-se ao sujeito da pesquisa o equipamento e o movimento que o mesmo deveria realizar, esclarecendo-o que não poderia realizar movimentos compensatórios. Em seguida, direcionou a cabeça primeiramente ao acrômio da escápula direita e, posteriormente à esquerda, mesmo procedimento realizado por Florêncio em 2010. ${ }^{12}$

Os dados apresentam-se como média e desvio padrão. Realizou-se primeiramente o teste de Kolmogorov-Smirnov. $O$ teste $T$ independente foi adotado para comparar as médias de ADM da coluna cervical dos dois grupos. Adotou-se nível de confiança com $\mathrm{p}<0,05$ para estes testes. Estes procedimentos foram realizados no software Graph Pad 3.0.1 (Graph Pad Insta, San Diego, CA, USA).

Esta pesquisa foi aprovada pelo Comitê de Ética em Pesquisa do Centro Universitário de João Pessoa/PB em 16 de Fevereiro de 2011, registro 1602/11. Todos os participantes da pesquisa foram solicitados a assinar o Termo de Consentimento Livre e Esclarecido de acordo com os princípios éticos estabelecidos na Resolução 196/96 ${ }^{13}$, vigente à época, do Conselho Nacional de Saúde que diz respeito às Diretrizes e Normas Regulamentadoras de Pesquisas Envolvendo Seres Humanos.

\section{RESULTADOS E DISCUSSÃO}

Os sujeitos do $\mathrm{Gl}$ possuíam $68 \pm 6$ anos, sendo $69 \%(\mathrm{n}=38)$ mulheres e os indivíduos do GJ $23 \pm 3$ anos de idade com $78 \%(n=43)$ mulheres. A Tabela 1 expõe média e desvio padrão da média das amplitudes lateral direita e esquerda da coluna cervical dos sujeitos.

Tabela 1- Representação da média e desvio padrão da média das amplitudes lateral direita e esquerda da coluna cervical dos sujeitos, João Pessoa/PB, 2011.

\begin{tabular}{lllll}
\hline Grupos & Amplitude lateral direita & Amplitude lateral esquerda \\
\hline & Média & Desvio padrão da média & Média & Desvio padrão da média \\
Idosos & $20.4^{*}$ & \pm 6.4 & $29^{*}$ & \pm 7 \\
Jovens & $31.1^{*}$ & \pm 7 & $32^{*}$ & \pm 6 \\
\hline
\end{tabular}

*Indica diferença estatística entre os dois grupos, $\mathrm{p}<0,05$.

Fonte: Dados das pesquisadoras, 2011.

Quanto à amplitude lateral direita da coluna cervical, registrou-se $20,4 \pm 6,4$ graus nos idosos estudados e $31,1 \pm 7$ graus nos jovens. A amplitude lateral esquerda revelou $29 \pm 7$ graus para idosos e $32 \pm 6$ graus nos jovens. Ao comparar as medidas das amplitudes, idosos e jovens apresentaram diferença estatística significativa $(p<0,05)$.

Os jovens apresentaram maior amplitude do movimento da coluna cervical, no entanto, ambos os grupos não atingiram o valor de amplitude estabelecido na literatura como $45^{\circ} .^{3}$ Contrapondo achados do estudo realizado em 2011 com 53 adultos, o qual detectou parâmetros dentro da normalidade e revelou que a dor não constitui-se fator limitante para amplitude de movimento cervical dos sujeitos avaliados. ${ }^{14}$

Considera-se que a dor interfere na qualidade de vida dos idosos, influenciando na sua fragilidade, afetando atividades de vida diária. Fato presente em estudo com 48 idosos no município de Cruzaltense/Rio Grande do Sul no ao 2009, onde 14,8\% responderam sentir dor na coluna cervical, observou-se ainda que muitos dos idosos convivam com a dor diariamente e evitavam relatar as dores por sentimentos de medo ou vergonha. ${ }^{15}$ 


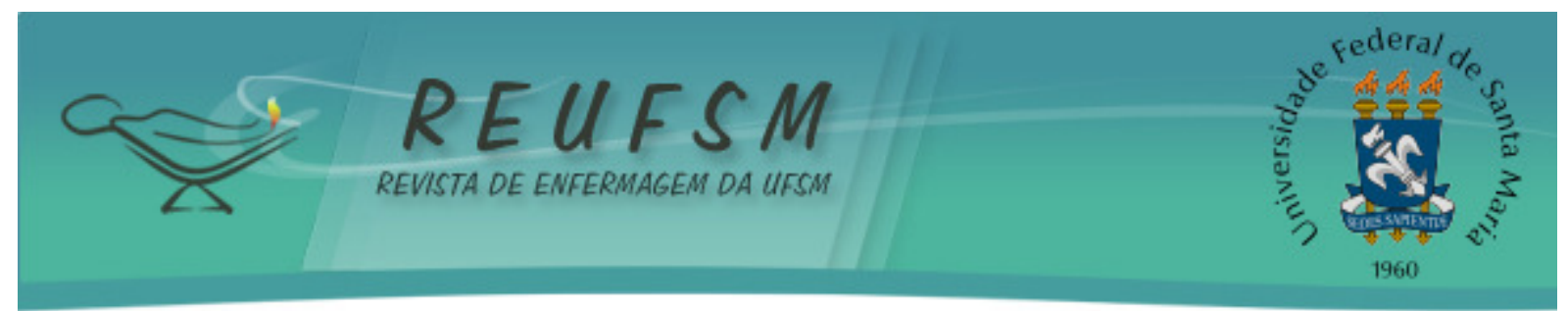

Voluntários idosos da Universidade Aberta da Terceira Idade (UATI) de São Carlos em $2010^{16}$, indagados quanto a incapacidade cervical afirmaram após intervenção de oficinas sobre cuidados na coluna, redução da intensidade da dor, principalmente, na região cervical, contudo, os resultados da pesquisa demostraram não ser suficientes para melhora na capacidade funcional da pessoa idosa.

Estudo ${ }^{17}$ realizado com jovens e idosos entre 20 a 78 anos determinou que o estilo de vida não possui influência no movimento de amplitude ativa, sendo observada nos testes: flexão, rotação e inclinação.

Júnior e colaboradores em estudo realizado em $2013^{18}$ destacaram carência da literatura referente a parâmetros na amplitude de movimento da coluna cervical, enfatizando a necessidade de realização de estudos futuros.

Confirmando os dados da pesquisa em Londrina/Paraná no ano $2011^{8}$, os idosos também apresentaram redução da amplitude do movimento, associado ao processo degenerativo do idoso, sejam eles articulares, ósseos ou musculares.

Para mensurar os graus de amplitude lateral da coluna cervical em diferentes faixas etárias deve-se considerar a existência da variabilidade natural, sobretudo nos idosos, em que o movimento da amplitude cervical decresce aproximadamente $5,5^{\circ}$ a $7,9^{\circ}$ por década. Todavia, não se tem definido na literatura valores padronizados do movimento da amplitude da coluna cervical para idosos. ${ }^{19}$

Considerando que os dois grupos não atingiram o valor de amplitude estabelecido na literatura para população geral como $45^{\circ 3}$, pode-se afirmar, em linhas gerais que ambos os grupos encontram-se em estado de alerta. Especificamente no grupo idoso é preciso considerar as características intrínsecas do processo de envelhecimento, no que consiste a diminuição de $5^{\circ}$ por década. Em outras palavras, comprova-se a necessidade de intervenção precoce de um profissional de saúde, em busca da prevenção ou diagnóstico prévio de lesões na coluna cervical.

\section{CONCLUSÕES}

Este estudo procurou conhecer as diferenças da amplitude lateral do movimento da coluna cervical entre jovens e idosos, identificando fragilidades no que concerne ao valor em graus ser inferior ao preconizado na literatura tanto para jovens como para idosos, sendo a diferença dessas medidas significativas entre os sujeitos dos dois grupos.

Como meio de diagnóstico de disfunções musculoesqueléticas faz-se necessário a avaliação da ADM da coluna cervical na busca de progressões de doenças e acompanhamento dos efeitos e evolução de pacientes.

0 aumento da idade favorece a diminuição da mobilidade da coluna cervical o que pode justificar o achado da pesquisa em lide, entretanto, os jovens também apresentaram redução da $A D M$, fato que pode simbolizar um alerta na perspectiva de descobrir as causas deste fenômeno.

Ressalta-se ainda que não existem valores padronizados de ADM da coluna cervical em idosos definidos na literatura. Sugere-se a criação de escalas voltadas para pediatria, adulto e idoso que possam avaliar periodicamente a ADM da coluna cervical em indivíduos que procurem atendimento de rotina em Unidades Básicas de Saúde, no intuito de minimizar futuros danos à vida destes indivíduos.

Nesse contexto, conclui-se ser necessário que os profissionais de saúde e gestores busquem estratégias para orientar a população quanto à importância da ADM da coluna cervical, o valor da amplitude e possíveis consequências atreladas a sua redução.

Para a enfermagem este estudo torna-se relevante, pois instiga novas condutas e protocolos no intuito de prevenir agravos, melhorando as condições de saúde da 


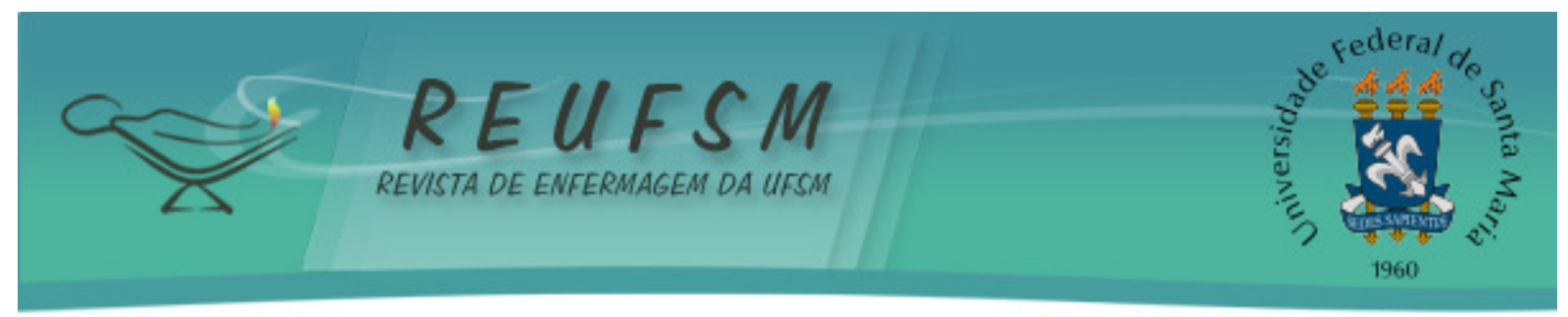

população. Remete-se à necessidade de criação de uma escala de medidas angulares condizente com a faixa etária dos indivíduos, podendo ser aferida pela equipe de enfermagem nas unidades básicas de saúde que se caracterizam como a principal porta de entrada para os serviços do Sistema Único de Saúde.

\section{REFERÊNCIAS}

1. Nakashima H, Yukawa Y, Ito K, Machino M, El Zahlawy H, Kato F. Posterior approach for cervical fracture-dislocations with traumatic disc herniation. Eur Spine J [Internet]. 2011 mar [acesso em 2014 abr 26];20(3):394-87. Disponível em: http://www.ncbi.nlm.nih.gov/pmc/articles/PMC3048228/.

2. Pilger C, Lentsck MH, Vargas G, Baratieri T. Causas de internação hospitalar de idosos residentes em um município do Paraná, uma análise dos últimos 5 anos. Rev Enferm UFSM [Internet]. 2011 set/dez [acesso em 2014 abr 27];1(3):394-402. Disponível em: http://cascavel.ufsm.br/revistas/ojs-2.2.2/index.php/reufsm/article/view/3186/2407.

3. Antônio SF, Pernambuco RA. Diagnóstico diferencial das cervicalgias. RBM Rev Bras Med [Internet]. 2014 abr [acesso em 2014 abr 27]. Disponível em: http: / / www.moreirajr.com.br/revistas.asp?fase=r003\&id_materia=1434.

4. Roberge RJ, Roberge MR. Overcoming barriers to the use of osteopathic manipulation techniques in the emergency department. West J Emerg Med. 2009;10(3):184-9.

5. Mansilla-Ferragut $P$, Fernández-de-Las-Penãs $C$, Albuquerque-Sendínin $F$, Cleland JA, Boscá-Gandía JJ. Immediate effects of atlanto-occipital joint manipulation on active mouth opening and pressure pain sensitivity in women with mechanical neck pain. $J$ Manipulative Physiol Ther. 2009;32(2):101-6.

6. Gelalis ID, DeFrate LE, Stafilas KS, Pakos EE, Kang JD, Gilbertson LG. Three-dimensional analysis of cervical spine motion: reliability of a computer assisted magnetic tracking device compared to inclinometer. Eur Spine J [Internet]. $2009 \mathrm{fev}$ [acesso em $2014 \mathrm{abr}$ 27];18(2):276-81. Disponível

http://www.ncbi.nlm.nih.gov/pmc/articles/PMC2899335/.

em:

7. Chaves TC, Nagamine HM, Belli JFC, Hannai MCT, Bevilaqua-Grossi D, Oliveira AS. Reliability of fleximetry and goniometry for assessing cervical range of motion among children. Rev Bras Fisioter. 2008;12(4):283-9.

8. Moreira MD, Marchiori LLM, Costa VSP, Damasceno EC, Gibrin PCD. Zumbido: possível associação com alterações cervicais em idosos. Arq Int Otorrinolaringol [Internet]. 2011 jul/ago/set [acesso em 2014 abr 25];15(3):333-7. Disponível em: http://www.scielo.br/pdf/aio/v15n3/v15n3a11.pdf. doi: 10.1590/S1809. 48722011000300011.

9. Carvalho EMS, Mota SPF, Silva GPF, Coelho Filho JM. A postura do idoso e suas implicações clínicas. Geriatria \& Gerontologia. 2011;5(3):170-4.

10. Brasil. Instituto Brasileiro de Geografia e Estatística (IBGE). Anuário Estatístico do Brasil. Rio de Janeiro (RJ): IBGE; 2010.

11. Peeler J, Anderson JE. Reliability of the Ely's test for assessing rectus femoris muscle flexibility and joint range of motion. J Orthop Res [Internet]. 2008 jun [acesso $2014 \mathrm{abr}$ 27];26(6):793-9. Disponível em: http://onlinelibrary.wiley.com/doi/10.1002/jor.20556/ abstract;jsessionid=41B145F0B9D425B5EB03EF56F47C4F97.f04t0. 


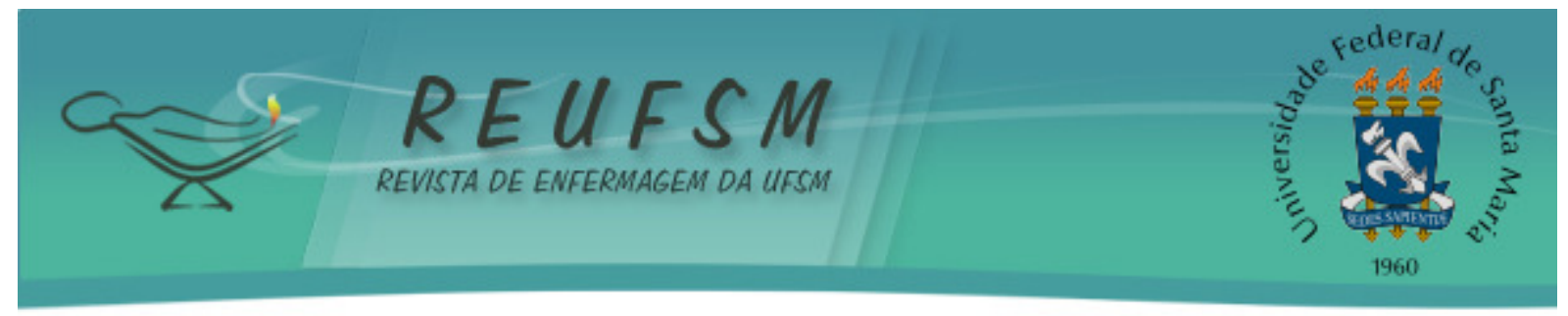

12. Florêncio LL, Pereira PA, Silva ERT, Pegoretti KS, Gonçalves MC, Bevilaqua-Grossi D. Concordância e confiabilidade de dois métodos não-invasivos para a avaliação da amplitude de movimento cervical em adultos jovens. Rev Bras Fisioter. 2010;14(2):175-81.

13. Brasil. Ministério da Saúde. Conselho Nacional de Saúde. Resolução CNS n²196, de 10 de outubro de 1996. Aprova diretrizes e normas regulamentadoras de pesquisas envolvendo seres humanos. Brasília: Ministério da Saúde, Conselho Nacional de Saúde; 1996.

14. Milanesi JM, Corrêa ECR, Borin GS, Souza JA. Pasinato F. Atividade elétrica dos músculos cervicais e amplitude de movimento da coluna cervical em indivíduos com e sem DTM. Fisiot Pesqui. 2011;18(4):317-22.

15. Celich KLS, Galon C. Dor crônica em idosos e sua influência nas atividades da vida diária e convivência social. Rev Bras Geriatr Gerontol. 2009;12(3):345-59.

16. Lobato DFM. Contribuições de um programa de Escola de Coluna a indivíduos idosos. RBCEH. 2010;7(3):370-80.

17. Smith K, Hall T, Robinson K. The influence of age, gender, lifestyle factors and subclinical neck pain on the cervical flexion-rotation test and cervical range of motion. Man Ther. 2008;13(6):552-9.

18. Bermudes Júnior E, Elias A, Melim R, Ribeiro DS, Mucciaccia SS, Frizera A, et al. Avaliação da amplitude de movimento da coluna cervical utilizando sensores inerciais: estudo piloto. Rev Bras Reabilitação e Atividade Física. 2013;2(1):45-51.

19. Lansade C, Laporte S, Thoreux P, Rousseau MA, Skalli W, Lavaste F. Three-dimensional analysis of the cervical spine kinematics: effect of age and gender in healthy subjects. Spine (Phila Pa 1976). 2009;34(26):2900-6.

Data de recebimento: $08 / 11 / 2013$

Data de aceite: 03/06/2014

Contato com autor responsável: Anna Cláudia Freire de Araújo Patrício

Endereço postal: Rua Joana Morais Lordão,76.Cristo.João Pessoa/PB.CEP 58071-650.

E-mail:claudia.freirearaujo@gmail.com 